\title{
Die ,Bestrickung' Hildebrand von Einsiedels Konfliktregulierung und Rechtsverständnis in einer kursächsischen Adelsfamilie im 17. Jahrhundert
}

\author{
von \\ CHRISTIAN HEINKER
}

\begin{abstract}
Als der kursächsische Adlige Hildebrand von Einsiedel der Ältere (1566-1647) auf Gnandstein Ende des Jahres 1641 an die Abfassung seines Testaments und die damit zusammenhängenden Verfügungen bezüglich seines Erbes ging, fand er für seinen jüngeren Sohn, Hildebrand von Einsiedel den Jüngeren (1598-1653), Formulierungen, die auf ein gestörtes Verhältnis schließen lassen. ${ }^{1}$ So wurde Hildebrand der Jüngere als ungehorsamer Sohn bezeichnet, dem jedoch in seiner väterlichen Disposition noch 20.000 Gulden ausgesetzt waren, die jeweils zur Hälfte auf den beiden schriftsässigen Rittergütern seines Vaters standen. (Teil-)Enterbungen unbotmäßiger Kinder waren auch in adligen Familien so ungewöhnlich nicht; in diesem Fall scheint das Zerwürfnis zwischen Vater und Sohn indessen exemplarisch gewesen zu sein.

Der Sohn Hildebrand befand sich zur Zeit der kurfürstlichen Bestätigung der väterlichen Verfügung bereits seit über drei Jahren in Haft auf der Festung Hohnstein ${ }^{2}$ und zwar auf massives Betreiben seines eigenen Vaters. Ein Zerwürfnis innerhalb einer Familie, das solche Folgen zeitigte, war wohl einzigartig im kursächsischen Adel und bedarf einer näheren Betrachtung. Dass Adlige wegen bestimmten Fehlverhaltens in Haft gehalten wurden, war an sich nichts Ungewöhnliches in einer Zeit, in der bereits Rang- und Ehrhändel leicht zu justitiablen Konflikten ausarten konnten. Traten hingegen innerhalb einer Familie Auseinandersetzungen auf, so wurden diese meist auch intern geregelt. Hingegen kam es in der Frühen Neuzeit durch zunehmende Verrechtlichung zu einer Institutionalisierung von Konflikten, was andererseits auch durch verstärkte Justiznutzung der Untertanen hervorgerufen wurde. Gerade die Gerichtsverfassung Kursachsens mit ihren Spruchkollegien sollte einen zunehmenden Einfluss auf die Rechtsprechung nehmen, die gleichwohl in Sachsen eine starke Bindung an den
\end{abstract}

1 Vgl. Sächsisches Staatsarchiv - Staatsarchiv Leipzig, Rittergut Gnandstein (im Folgenden: StA Leipzig, RG Gnandstein), (Urkunden), U 119, Dresden, 24. November 1641, unterschrieben vom Kanzler und Geheimrat Heinrich von Friesen, der u. a. auch als Zeuge fungierte, und dem Kurfürsten selbst. Die Urkunde hatte auch die Funktion einer kurfürstlichen Verleihung der auf den Rittergütern Gnandstein und Wolftitz stehenden Summe.

2 Festungshaft galt noch als Privileg adliger Delinquenten, die im Kurstaat in der Regel auf den Festungen Sonnenstein, Stolpen, Königstein, Hohnstein und zuweilen auch auf der Leipziger Pleißenburg einsaßen. Der Königstein galt schon im 16. Jahrhundert als sicherste Festung, u. a. wurde der unter Kurfürst Christian I. mächtige Kanzler Krell dort (15911601) bis zu seiner Hinrichtung gefangen gehalten. 1639 schlug Hildebrand der Ältere dem Kurfürsten vor, seinen Sohn von Hohnstein auf den Königstein setzen zu lassen, beÿ kegenwertigen gefäbrlichen leüfften sei dieser desto gewißer zu balten. Im gleichen Jahr widerstand Hohnstein indes einer schwedischen Belagerung; vgl. Sächsisches Staatsarchiv Sächsisches Hauptstaatsarchiv Dresden (im Folgenden: HStA Dresden), Loc. 7193/6, fol. 147r-149v, Gnandstein, 30. Juni 1639, Hildebrand von Einsiedel der Ältere an den Kurfürsten. 
Landesherrn aufwies und damit eng in die kursächsische Behördenorganisation integriert war. ${ }^{3}$ Der Kurfürst dominierte nach wie vor als oberster Gerichtsherr, sofern er diese Rolle anstrebte.

Tatsächlich war das Verhältnis des Vaters Hildebrand von Einsiedel zu seinem Sohn über die Jahre in immer stärkerer Weise von Entfremdung gekennzeichnet. In ihrer Mentalität müssen beide wohl als Antipoden betrachtet werden, die eine jeweils grundsätzlich differierende Auffassung vom Leben und der Rolle des Einzelnen darin besaßen.

\section{Der familiäre Hintergrund - Die Einsiedels als bedeutende Säule des Kurstaates}

Schon seit Ausgang des Mittelalters müssen die Einsiedels als eine der wichtigsten Adelsfamilien des werdenden Fürstenstaates betrachtet werden. Dies bezog sich sowohl auf die Erwerbsstrategie von Grundbesitz und Rittergütern als auch auf die Nähe zu den sächsischen Herzögen und Kurfürsten, was sich zuvorderst in vielfältigen Ratsdiensten niederschlug. Als Familie seit etwa 1409 auf Gnandstein ansässig, ist zunächst Hildebrand von Einsiedel (um 1400-1461) als Obermarschall des sächsischen Kurfürsten nachzuweisen. In der Folgezeit waren mehrere Einsiedels als vertraute Räte der sächsischen Kurfürsten anzutreffen, so Heinrich I. (1435-1507), ${ }^{4}$ vor allem aber Heinrich Hildebrand (1497-1557), der als Rat für fünf sächsische Kurfürsten fungierte und als Freund Luthers als einer der ersten Adligen Sachsens zum neuen Glauben übertrat. ${ }^{5}$ Mit dessen Sohn Hildebrand II. (1528-1598) fand jedoch die Ratstradition der Gnandsteiner Linie zunächst ihr Ende.

Hinsichtlich ihres Güterbesitzes fallen die Einsiedels seit dem 15. Jahrhundert durch eine umsichtige und kluge Strategie sowohl des Erwerbs als auch des Haltens ihrer Rittergüter auf. ${ }^{6}$ Auffällig war weiterhin eine relativ geschlossene Form des Besitzes zunächst vornehmlich im Kohrener Land ohne die in anderen Familien durchaus auftretende Zersplitterung. Bis weit ins 19. Jahrhundert besaß diese Adelsfamilie die meisten und wohl einträglichsten Rittergüter des Leipziger Kreises und darüber hinaus auch Sachsens. ${ }^{7}$ Weiterhin wurden die Rittergüter der Einsiedels

3 Vgl. HeINER LÜCK, Die Spruchtätigkeit der Wittenberger Juristenfakultät. Organisation - Verfahren - Ausstrahlung, Köln/Weimar/Wien 1998.

4 Dieser hatte einen deutlichen Einfluss auf die Leipziger Teilung 1485 zwischen Ernestinern und Albertinern. Die Grenzziehung verlief durch die Einsiedelschen Besitzungen; so blieben Mitglieder dieser Familie beiden Fürsten als vertraute Räte erhalten; vgl. auch FAlK SCHUlze, Die Herren von Gnandstein - 800 Jahre Geschichte und Geschichten einer sächsischen Burg und ihrer Besitzer, in: Simona Schellenberger (Hg.), Burg Gnandstein, Leipzig 2000, S. 73-90, hier S. 78.

5 Vgl auch: Jens KunZE, Die Testamentsstiftung des Heinrich Hildebrand von Einsiedel - Beginn einer Familientradition, in: Die Familie von Einsiedel. Stand, Aufgaben und Perspektiven der Adelsforschung in Sachsen (Kolloquium des Sächsischen Staatsarchivs/ Staatsarchiv Leipzig in Zusammenarbeit mit der Universität Leipzig 9. November 2005), hrsg. vom Sächsischen Staatsarchiv Leipzig, Leipzig 2007, S. 92-100.

6 Bereits im 15. Jahrhundert zählten die Einsiedels auch nach dem Steueraufkommen zu den wirtschaftlich potentesten Adelsfamilien Sachsens; vgl. HeLGa REICH/BIRGIT RicHTER, Besitzgeschichte sowie Archivbildung bei der Familie von Einsiedel und die Überlieferung im Staatsarchiv Leipzig, in: Die Familie von Einsiedel (wie Anm. 5), S. 53-65, hier S. 58 .

7 Eine Aufstellung über den Besitz der adligen Geschlechter von 1681 bis 1844 sieht die Einsiedels in jedem Jahr hinsichtlich der Zahl der Rittergüter im Leipziger Kreis in der 
zumeist schriftsässig gehalten. Dies hatte u. a. den Vorteil, dass im Falle von Konflikten direkt mit dem Landesherrn kommuniziert werden konnte, ohne den ,Umweg' über das Amt. Die Einsiedels verfügten damit über ein direktes Verhältnis zum Kurfürsten und zu dessen Zentralbehörden.

Die ältere Gnandsteiner Linie, die von Hildebrand II. begründet worden war, verfügte über die altschriftsässigen Rittergüter Prießnitz, Wolftitz und Gnandstein. Als ältestem Sohn Hildebrands II. fielen Hildebrand III. (1566-1647) nach dem Tode seines Vaters Gnandstein und Wolftitz zu. Hildebrand III. besaß von Beginn an ein biografisch fassbares, besonderes Verhältnis zum alten Herkommen seiner Familie, das sowohl von Stolz und Würde hinsichtlich der Familientradition als auch von eher theologisch grundiertem Rechtsverständnis geprägt war. Schon während seines Studiums in Tübingen ${ }^{8}$ ließ er erkennen, dass für ihn eher theologische Aspekte im Vordergrund standen, obwohl auch Politica und Jurisprudentia zu seinen Studien zählten. ${ }^{9}$ Hier lernte er den zunehmenden Drang nach konfessioneller Homogenität der lutherischen Landesherren kennen, als während seines Studiums 1582 in Tübingen der Eid auf die Konkordienformel, der jegliche religiöse Indifferenz fortan ausschließen sollte, für Professoren und ,Universitätsverwandte' obligatorisch wurde. ${ }^{10}$

In gewisser Weise befolgte auch Hildebrand III. die Familientradition der Einsiedels; er wurde Obersteuereinnehmer wie sein Vater. Hinsichtlich seiner persönlichen Lebensgestaltung ist jedoch die theologische Fundierung unübersehbar. Schon sein Testament von 1626 übergab er der Theologischen Fakultät der Universität Leipzig zur Zensur ${ }^{11}$ und forderte ein Gutachten ein; ein auch im 17. Jahrhundert eher ungewöhnliches Verhalten. Weiterhin ließ Hildebrand III. als Verfasser diversen theologischen Schrifttums erkennen, wie sehr ihm der wahre lutherische Glauben am Herzen lag. ${ }^{12}$ Auch um die Visualisierung der Familientradition machte sich Hildebrand ver-

ersten Reihe; vgl. Axel FlÜGEL, Bürgerliche Rittergüter. Sozialer Wandel und politische Reform in Kursachsen (1680-1844) (Bürgertum. Beiträge zur europäischen Gesellschaftsgeschichte 16), Göttingen 2000, S. 273.

8 Matrikeleintrag von Hildebrand, der 1581 mit seinem zwei Jahre älteren Bruder Heinrich zum Studium nach Tübingen kam; vgl. Heinrich Hermelink (Hg.), Die Matrikeln der Universität Tübingen, Bd. 1: 1477-1600, Stuttgart 1906, S. 594, Nr. 30 und 31 vom 5. Juni 1581.

9 Biografische Angaben auch nach: Curriculum Vitae Hildebrandi ab Einsiedel in Gnandstein und Wolfftitz 1647, vgl. StA Leipzig, RG Gnandstein, Nr. 840.

10 Vgl. Klaus Schreiner, Disziplinierte Wissenschaftsfreiheit. Gedankliche Begründung und geschichtliche Praxis freien Forschens, Lehrens und Lernens an der Universität Tübingen (1477-1945), Tübingen 1981, S. 13. Die religiöse Einheitlichkeit führte mittelfristig zu einer Provinzialisierung Tübingens. Mit hoher Wahrscheinlichkeit wurde Hildebrand III. von Einsiedel vom Professor der Theologie, Jakob Heerbrand (1521-1600), beeinflusst, der noch bei Luther und Melanchthon gehört hatte und seit 1557 in Tübingen lehrte; vgl. ein Kurzporträt Heerbrands in: Hansmartin Decker-HaufF/WiLfried SETZler (Hg.), Die Universität Tübingen von 1477 bis 1977 in Bildern und Dokumenten, Tübingen 1977, S. 92 f. Für die kursächsische Universitätsreform von 1580 konnte Tübingen z. T. zum Vorbild avancieren; vgl. MANFRED RUDERSDORF, Tübingen als Modell? Die Bedeutung Württembergs für die Vorgeschichte der kursächsischen Universitätsreform von 1580, in: Armin Kohnle/Frank Engehausen (Hg.), Zwischen Wissenschaft und Politik. Studien zur deutschen Universitätsgeschichte. Festschrift für Eike Wolgast zum 65. Geburtstag, Stuttgart 2001, S. 67-85.

11 Vgl. StA Leipzig, RG Gnandstein, Nr. 840.

12 Ein Verzeichnis seiner Schriften, die eine tiefe theologische Fundierung erkennen lassen, in: StA Leipzig, RG Gnandstein, Nr. 841 (z. B. De Vocatione Ecclesiastica, und 
dient. Auf sein Bemühen, das alte Herkommen und die familiäre Würde der Einsiedels auch künstlerisch darzustellen, gehen die heute noch zu bewundernden Epitaphien in der Gnandsteiner Dorfkirche zurück. ${ }^{13}$ Frömmigkeit und Herrschaftsmanifestation spiegelten sich in Ausgestaltung der Kirchen und in Altarstiftungen. ${ }^{14}$ Die Aktivitäten und die Lebensweise Hildebrands III. von Einsiedel fügten sich damit ein in die verstärkten Bemühungen auch des sächsischen Adels zur Statussicherung. ${ }^{15}$

\section{Der wachsende Konflikt zwischen Vater und Sohn}

Vor diesem Hintergrund musste der Gegensatz Hildebrands des Älteren zum Lebenswandel seines gleichnamigen Sohnes besonders harsch ausfallen. Hildebrand von Einsiedel der Jüngere zeigte so gar keine Ambitionen zu studieren ${ }^{16}$ und widmete sich lieber dem Kriegshandwerk, bei dem er es immerhin bis zur Charge eines ObristWachtmeisters und Majors brachte. Von 1620 datieren dagegen erste Schuldforderungen an ihn, die er offenkundig allzu leichtfertig unterschrieben hatte. ${ }^{17}$

In seiner väterlichen Disposition von 1626, die die Funktion eines Testaments besaß, formulierte Hildebrand der Ältere dann erste Sanktionen, die deutlich machten, dass er den Lebenswandel seines zweitgeborenen Sohnes ${ }^{18}$ missbilligte. Er setzte diesem zwar 20.000 Gulden aus, jedoch mit der Auflage: doch dergestalt und anders nicht abgestattet werden, es würde denn Hildebrandt sich käufflich einlassen, und ein stück Lehnguts an sich, die gebrüder und neheste Agnaten aber, neben mir, in die gesambte hand und mit belehnschafft bringen, und solch gutt wircklich übernehmen, und

Beruff der Kirchendiener; Christliches Gebet, auff die itzige, Letzte, betrückt Zeit gerichtet; Gebeth und Sterbe-Kunst, Valetsegen und eines wabren Christen Recht Adeliche und Geistliche Abnen, Schilder, Wapen, und Waffen).

13 Mitten im Dreißigjährigen Krieg begann um 1640 Hildebrand der Ältere mit der Grablege seiner Familie, vgl. SCHUlzE (wie Anm. 4), S. 84.

14 Zur lutherischen Frömmigkeit im Adelshaus Einsiedel, die stark ausgeprägt war; vgl. MARIUS WINZELER, Burgkapelle, Patronatskirche, Familiengrablege - Tradition und Wandel der Adelsfrömmigkeit und ihres künstlerischen Ausdrucks im 16. und 17. Jahrhundert. Das Beispiel der Familie von Einsiedel, in: Katrin Keller/Josef Matzerath (Hg.), Geschichte des sächsischen Adels, Köln/Weimar/Wien 1997, S. 207-224.

15 Vgl. Gernot Heiss, Habitus, Bildung und Familie - Strategien des Adels zur Statussicherung, in: Keller/Matzerath, Geschichte des sächsischen Adels (wie Anm. 14), S. 321-326.

16 Sein Präzeptor deutete schon 1616 seiner Mutter gegenüber an, dass er nicht studieren wolle; Hildebrand habe seinen Bruder gebeten, dass es der Vater nicht erfahren möchte; vgl. HStA Dresden, Loc. 7193/10, fol. 76v. In den Matrikeln der Universität Leipzig ist Hildebrand von Einsiedel der Jüngere nicht nachzuweisen. In einem Schreiben vom 9. Februar 1616 aus Leipzig schrieb der Präzeptor Georg Hagen nach Gnandstein, Junker Hildebrand habe keine adeligen rühmlichen Tugenden; er zweifele an seiner Besserung leider nicht wenig, es sei fürwahr höchlich zu beklagen, dass er so degeneriert sei; vgl. HStA Dresden, Loc. 7193/6, fol. 61r.

17 Vgl. StA Leipzig, RG Gnandstein, Nr. 1288, Schuldforderungen des Lorenz Werner, Dresden, an Hildebrand von Einsiedel den Jüngeren, 1620/21. 1620 hatte der Fähnrich Hildebrand von Einsiedel einen Schuldbrief mit eigenen Händen unterschrieben und mit eigenem Petschaft besiegelt.

18 Hildebrand der Ältere hatte insgesamt fünf Söhne, von denen zwei früh verstorben waren. In den 1620er-Jahren lebten noch: Heinrich (1592-1649), Hildebrand und Alexander (1601-1637). 
beziehen. ${ }^{19}$ Die Rittergüter Gnandstein und Wolftitz sollten hingegen jeweils an den älteren und jüngeren Bruder Hildebrands fallen. Dass bei drei erbberechtigten Söhnen einer leer ausgehen bzw. mit Geld abgefunden werden musste, war angesichts der Tatsache, dass die Lehngüter nicht zerteilt werden konnten und sollten, einleuchtend. Ähnliche Verfügungen waren im Hause Einsiedel durchaus schon üblich gewesen. ${ }^{20}$ Im Falle Hildebrand von Einsiedels war die väterliche Disposition jedoch als Mahnung und Warnung an seinen Sohn zu verstehen, seinen Lebenswandel der Würde und Tradition seiner Familie anzupassen.

Der Sohn hingegen lebte offenkundig dauerhaft über seine Verhältnisse. Als er 1627/28 doch noch an der Universität Straßburg21 aufzufinden war, musste er seine Brüder brieflich beschwichtigen, dass er sein Geld nicht durchgebracht habe. ${ }^{22} \mathrm{Im}$ Jahr 1630 musste Hildebrand der Ältere seine Söhne jedoch anweisen, ihrem Bruder unter keinen Umständen Schuldverschreibungen auszuhändigen und diesen an gegebene Versprechen zu erinnern. Immerhin erreichte der Vater, dass sein leichtfertiger Sohn schriftlich in einem Revers versprach, sich zu bessern. ${ }^{23}$

Die im gleichen Jahr erfolgte brüderliche Disposition warf indes ein Schlaglicht auf die wahren Verhältnisse und das Misstrauen, das Hildebrand von seinen Familienmitgliedern entgegengebracht wurde. Heinrich und Alexander sollten sich im Falle des Todes wechselseitig beerben, während Hildebrand mit barem Geld abgefunden werden sollte. ${ }^{24}$

19 Väterliche Disposition vom 31. Oktober 1626, vom Kurfürsten bestätigt 5. Januar 1627; vgl. StA Leipzig, RG Gnandstein, Nr. 754. Zum Wert von Testamenten als sozialgeschichtliche Quelle: WIELAND HELD, Frühneuzeitliche Testamente sächsischer Landadliger, ihr bisher unterschätzter Wert als sozial- und wirtschaftsgeschichtliche Quellen, in: Leipzig, Mitteldeutschland und Europa. Festgabe für Manfred Straube und Manfred Unger zum 70. Geburtstag, hrsg. im Auftrag des Leipziger Geschichtsvereins von Hartmut Zwahr/Uwe Schirmer/Henning Steinführer, Beucha 2000, S. 349-356.

20 So wurde bei einem Erbvergleich der Söhne Heinrich Hildebrand von Einsiedels (1497-1557) im Jahr 1561 Haubold mit Geld abgefunden, das in unterschiedlicher Höhe als Ausgleichszahlung von den Rittergütern der anderen Söhne erfolgte; vgl. KunZE, Testamentsstiftung (wie Anm. 5), S. 98.

21 Sein Matrikeleintrag findet sich unter den Studierenden der Jurisprudenz; vgl. GustaV C. KNOD (Bearb.), Die Alten Matrikeln der Universität Straßburg 1621-1793, Bd. II: Die Matrikeln der Medicinischen und juristischen Facultät (Urkunden und Akten der Stadt Straßburg, hrsg. mit Unterstützung der Landes- u. der Stadt-Verwaltung, III. Abtheilung: Die Alten Matrikeln der Univ. Straßburg), Straßburg 1897, S. 218, 8. Oktober 1627, Nr. 64.

22 In einem Schreiben von Januar 1628 an seine Brüder Heinrich und Alexander berichtet Hildebrand der Jüngere, dass er sein Geld an Kleidung und anderen Sachen haben müsse. Zur Finanzierung führte er die Jahreszinsbescheinigung seiner Brüder an; vgl. HStA Dresden, Loc. 7193/11, fol. 5 f., 14. Januar 1628.

23 Vgl. StA Leipzig, RG Gnandstein, Nr. 1289, Liederlicher Lebenswandel und Schuldenwesen Hildebrand d. J. 1630, 1633, 1637, fol. 10. Dort auch ein von Hildebrand dem Älteren und seinen beiden Söhnen gegen Hildebrand gerichteter unterschriebener und gesiegelter Revers vom 2. September 1630. Darin führte der Vater aus, dass sein Sohn Hildebrand geldes fast unersetig ist. Er habe vielleicht seine tief eingebildete reputation, und vermeinte hoheit desto beharlicher naußzufübren.

24 Vgl. Brüderliche Disposition zwischen Heinrich, Hildebrand und Alexander vom 7. Juli 1630. Hildebrand sollte danach mit 4.000 Gulden innerhalb von drei Jahren abgefunden werden. Er solle gebührlich quittieren. Der Wert der beiden Rittergüter Gnandstein und Wolftitz wurde dabei mit zusammen 60.000 Gulden angeschlagen; vgl. StA Leipzig, RG Gnandstein, Nr. 754, unpag. 
1635 sah sich Hildebrand der Ältere jedoch gezwungen, in einer zusätzlichen Erklärung zu seinem Testament den offenkundigen Mangel an Besserung seines Sohnes Hildebrand noch einmal gebührend herauszustellen und dessen Verfehlungen einzeln anzuprangern. 25

So warf Hildebrand der Ältere seinem Sohn Schuldenmacherei, Verschwendung, üble Nachrede, arglistige Täuschung und Urkundenfälschung vor;26 Tatbestände, die den Vater in ihrer Summe noch einmal bestärkten, die Verfügungen seines Testaments hinsichtlich seines gleichnamigen Sohnes zu bekräftigen. 1637 erreichten die Missstimmungen Hildebrands des Älteren die Dresdner Regierungsebene, als der Kanzler in einem Schreiben an den Schösser von Borna durchblicken ließ, wie sehr Hildebrand sich über seinen Sohn beklagt habe. ${ }^{27}$

Einen neuen Höhepunkt erreichte das Zerwürfnis zwischen Vater und Sohn Anfang 1638, als Hildebrand der Jüngere bei seinem Bruder auf Wolftitz zu Gast war. Nach einem Disput, bei dem Hildebrand es seinem Bruder Heinrich übel vermerkte, dass er nicht zum Begräbnis des anderen Bruders Alexander ${ }^{28}$ geladen war, stieß Hildebrand der Jüngere üble Verwünschungen gegen seinen Vater aus. ${ }^{29}$ Als dem Vater diese massiven Ehrverletzungen zu Ohren kamen, war das Maß für ihn offenkundig voll. ${ }^{30}$ Hildebrand der Ältere setzte sich nun beim Kurfürsten persönlich dafür ein, dass sein

25 Zusätzliches, seinem Testament beigefügtes Schriftstück vom 18. August 1635: Kurzer Anhang und fernere Erklärung meiner väterlichen Disposition, Mebrentlich meinem übell verfübreten Sohn Hildebranden betreffend; vgl. StA Leipzig, RG Gnandstein, Nr. 754. Jede einzelne Seite ist dabei namentlich abgezeichnet.

26 So habe Hildebrand der Jüngere beinahe 12.000 Gulden Schulden gemacht und diverse Schuldscheine im Namen seines Vaters und seiner Brüder unterschrieben; vgl. StA Leipzig (wie Anm. 25).

27 Vgl. StA Leipzig, RG Gnandstein, Nr. 1290. In einem Schreiben vom 11. Dezember 1637 berichtet Kanzler Wolf von Lüttichau dem Schösser von Borna, Esaias Hauenstein, dass sich Hildebrand von Einsiedel über seinen Sohn beklage wegen dessen gottlosen Lebens, ärgerlichen Wandels und Widerwillens sowie Ungehorsam gegen seinen Vater.

28 Alexander von Einsiedel war im September 1637 gestorben, sodass von den ursprünglich fünf Brüdern nun noch zwei am Leben waren.

29 Vgl. StA Leipzig, RG Gnandstein, Nr. 1290, Zeugnis - Register Hildebrand von Einsiedel des Älteren (Gnandstein) contra Hildebrand von Einsiedel des Jüngeren in Haft gehaltenen Sohnes vom 9. März 1640. Nach Zeugenaussagen sei Hildebrand der Jüngere am 4. Januar 1638 bei seinem Bruder Heinrich zu Gast gewesen und habe ihn gefragt, warum der Vater ihn beim Kurfürsten verklagt habe und warum er nicht zum Begräbnis des Bruders Alexander gebeten worden wäre. Darauf habe Heinrich geantwortet: Das wüsste er nicht. Er habe damit nichts zu tun. Daraufhin habe Hildebrand der Jüngere gesagt, Donner und Blitz sollen dem Alten ins Herze fahren und ihn in die Erde hinein schlagen.

30 Vgl. Klaus Schreiner/Gerd Schwerhoff (Hg.), Verletzte Ehre. Ehrkonflikte in Gesellschaften des Mittelalters und der Frühen Neuzeit (Norm und Struktur 5), Köln/Weimar/Wien 1995. Zu beachten ist, dass verletzte Ehre keineswegs nur ein Elitenphänomen darstellt. Da der Ehrbegriff mithin eine sakrale Wurzel besitzt, musste die Fallhöhe der empfundenen Ehrverletzung bei Hildebrand dem Älteren besonders groß ausfallen. Wie hoch diese Verbalinjurien angebunden wurden, zeigte ein Bibelzitat im gleichen Juramentum Testis: Gott sagt, wer Vater und Mutter flucht, der soll des Todes sterben. Zu Injurien auch: RALF-PETER FUCHS, Ehrkämpfe. Injurienprozesse in der Frühen Neuzeit und ihre Interpretationsmöglichkeiten, in: Rheinisch-westfälische Zeitschrift für Volkskunde 42 (1997), S. 29-50. 
Sohn in dauernde Haft komme. ${ }^{31}$ Hildebrand der Jüngere hielt sich mittlerweile häufig außerhalb Kursachsens auf, sodass der Kurfürst seinen Aufenthaltsort ermitteln ${ }^{32}$ und an Herzog Johann Philipp von Sachsen-Altenburg ein Auslieferungsersuchen stellen musste. ${ }^{33}$

Hildebrand von Einsiedel bedankte sich sogleich beim Kurfürsten für die prompte Umsetzung seines Wunsches; ${ }^{34}$ dieser hielt ihn jedoch an, die Gründe schriftlich einzureichen, warum er seinen Sohn in ewiger Haft halten solle. Hildebrand von Einsiedel der Ältere sah offenbar keine andere Möglichkeit, seinen devianten Sohn dauerhaft disziplinieren zu können.

\section{Sozialdisziplinierung und Kriminalitätsforschung}

Die Geschichtsforschung zur Frühen Neuzeit ist seit 1969 durch das Paradigma der Sozialdisziplinierung wesentlich mitgeprägt worden. Für den Verfassungshistoriker Gerhard Oestreich war Sozialdisziplinierung das entscheidende Ergebnis des monarchischen Absolutismus. ${ }^{35}$ In der Folgezeit sollte sich dieses Forschungskonzept einer breiten Rezeption erfreuen; nicht zuletzt ließen sich die Erforschung frühneuzeitlicher Kriminalität und die Etablierung einer frühmodernen Gerichtsverfassung gut in dieses Modell einordnen. Tatsächlich erfreut sich das Konzept der Sozialdisziplinierung heute noch innerhalb der Verfassungsgeschichte einer gewissen Wertschätzung, ${ }^{36} \mathrm{da}$ es, eher vom institutionalistischen Ansatz ausgehend, an der (juristischen) Norm orientiert ist.

In der Sozialgeschichte ließ dagegen seit den Neunziger Jahren die Prägekraft dieses Konzeptes spürbar nach. Öffnete sich die Forschung nach und nach auch kulturanthropologischen Fragestellungen, so wurde schon bald deutlich, dass das Konzept Sozialdisziplinierung einen primär etatistischen Ansatz verfolgte und auch im Zuge der Ausweitung des Forschungsparadigmas auf andere Zeiträume unschärfer wurde. ${ }^{37}$

31 In einem siebenseitigen Brief vom 1. April 1638 setzte Hildebrand der Ältere dem Kurfürsten die Verfehlungen seines Sohnes noch einmal auseinander und bat ihn, an seinem Sohn die angedrohte Gefängnisstrafe für die Zeit seines ganzen Lebens zu vollstrecken; vgl. HStA Dresden, Loc. 7193/6, fol. 1r-4r. Die Verbalinjurien des Sohnes gegen seinen Vater wurden noch einmal hervorgehoben.

32 Am 10. April 1638 gingen Schreiben des Kurfürsten an den Schösser zu Chemnitz und Herzog Johann Philipp zu Sachsen-Altenburg ab; vgl. HStA Dresden, Loc. 7193/6, fol. $5 \mathrm{f}$. Schon eine Woche später vermeldete Herzog Johann Philipp, dass Hildebrand verhaftet und aufs Rathaus gebracht worden sei; vgl. ebd., fol 7.

33 Schreiben des sächsischen Kurfürsten vom 22. April 1638 an Herzog Johann Philipp zu Sachsen-Altenburg gute Aufsicht über den Gefangenen zu haben, dass dieser u. a. nicht mit seinem Anhang korrespondieren möge.

34 Hildebrand von Einsiedel auf Gnandstein, 26. April 1638. U. a. versicherte Hildebrand dem Kurfürsten, für alle Unkosten, die sein Sohn verursache, aufzukommen; vgl. HStA Dresden, Loc. 7193/6, fol. 9 f. Zum kurfürstlichen Wunsch, die Haftgründe schriftlich einzureichen vgl. ebd., fol. $16 \mathrm{f}$.

$35 \mathrm{Vgl}$. Gerhard Oestreich, Strukturprobleme des europäischen Absolutismus, in: Ders., Geist und Gestalt des modernen Staates. Ausgewählte Aufsätze, Berlin 1969, S. 179197.

36 Vgl. Dietmar Willoweit (Hg.), Deutsche Verfassungsgeschichte. Vom Frankenreich bis zur Wiedervereinigung Deutschlands, München ${ }^{5} 2005$.

37 So nahm Heinz Schilling unter weitgehender Akzeptanz das Modell der Sozialdisziplinierung auch für das konfessionelle Zeitalter in Anspruch, wodurch es allerdings an 
Beiden Ansätzen, dem Konzept der Sozialdisziplinierung als auch seinen Kritikern, ist eine zumindest partielle Fixierung auf die Makrostruktur "Staat“ zu eigen. ${ }^{38}$

In der gleichen Weise, wie das Konzept Sozialdisziplinierung an Rezeption verlor, vollzog sich der Aufstieg der Kriminalitätsgeschichte, die seit den Neunziger Jahren zu einem fest etablierten Bestandteil nicht nur der Frühneuzeitforschung geworden ist. ${ }^{39}$ Hierbei kamen mehr und mehr mentalitätsgeschichtliche und kulturanthropologische Fragestellungen ${ }^{40}$ zum Tragen, da in Ergänzung eines bisher eher institutionalistisch betriebenen Ansatzes zunehmend der Mensch in seiner möglichen Devianz und Delinquenz im Zentrum der Forschung stand. Seitdem stehen eher Fragen im Mittelpunkt des Forschungsinteresses, die die Kriminalität und die gesellschaftliche Entwicklung gemeinsam betrachten, womit sich Phänomene wie gesellschaftlicher Wandel besser als bisher erklären lassen. Im Ganzen steht oder fällt das Konzept der Sozialdisziplinierung mit der Frage, ob deviantes Verhalten auf einen Mangel an Triebkontrolle zurückzuführen oder möglicherweise als Ausdruck eines alternativen sozialen Codes der Ehre zu betrachten ist. ${ }^{41}$ Nach dieser Auffassung wäre eher das soziale Milieu der Normgeber und die Sozialdisziplinierung hätte damit die Funktion einer Dämpfung der Affekte.

Im Ganzen lässt sich die Frage nach Devianz oder Disziplinierung wohl noch am ehesten im Rahmen der Mentalitätsgeschichte unterbringen, da hier der Mensch als handelndes oder nichthandelndes Subjekt im Mittelpunkt steht und so abseits der Institutionen wie Gerichte und Spruchkollegien in der Analyse strafrechtlicher Sanktionen auch Phänomene wie soziale Kontrolle und Konfliktregulierung angemessen berücksichtigt werden können.

\section{Die Haft Hildebrand von Einsiedels - Die Urteile der Spruchkollegien}

Als Hildebrand von Einsiedel der Jüngere im Mai 1638 auf dem Hohnstein in Verwahrung genommen wurde, konnte er noch nicht ahnen, dass dies den Auftakt zu einer fast fünfjährigen Festungshaft darstellen sollte. In der Folgezeit entwickelte sich ein reger Schriftwechsel sowohl zwischen Hildebrand dem Älteren und dem Kurfürsten als auch zwischen Hildebrand dem Jüngeren und seinem Landesherrn.

Kontur verloren hat; vgl. HeINZ SCHILLING (Hg.), Kirchenzucht und Sozialdisziplinierung im frühneuzeitlichen Europa, Berlin 1994.

38 Oestreich postulierte zwar einen die Gesellschaft umfassenden Mentalitätswandel als epochemachendes Phänomen, Bezugsgröße dabei blieb bei ihm freilich der (absolutistische) Staat als Motor dieser Entwicklung.

39 Aus der mittlerweile kaum noch zu überschauenden Masse an Veröffentlichungen exemplarisch: ANDREAS BlauerT/GERD SCHWERHOFF (Hg.), Kriminalitätsgeschichte. Beiträge zur Sozial- und Kulturgeschichte der Vormoderne, Konstanz 2000; GERD SCHWERHOFF, Aktenkundig und gerichtsnotorisch. Einführung in die Historische Kriminalitätsforschung, Tübingen 1999; MARK HÄBERleIN (Hg.), Devianz, Widerstand und Herrschaftspraxis in der Vormoderne. Studien zu Konflikten im südwestdeutschen Raum (15.-18. Jahrhundert), Konstanz 1999; REBEKKA HABERMAS/GERD SCHWERHOFF (Hg.), Verbrechen im Blick. Perspektiven der neuzeitlichen Kriminalitätsgeschichte, Frankfurt am Main 2009.

40 Vgl. JOACHIM EIBACH, Kriminalitätsgeschichte zwischen Sozialgeschichte und Historischer Kulturforschung, in: Historische Zeitschrift 263 (1996), S. 681-715.

$41 \mathrm{Zu}$ dieser Gegenüberstellung vgl. SusAnNa BurghaRTZ, Disziplinierung oder Konfliktregelung? Zur Funktion städtischer Gerichte im Spätmittelalter. Das Züricher Ratsgericht, in: Zeitschrift für Historische Forschung 16 (1989), S. 385-409. 
Was die Frage der juristischen Zuständigkeit anging, so war die Landesregierung mit dem Kanzler an der Spitze erste Anlaufstelle, wenn es um Kriminal- und Verwaltungsprozesse ging. ${ }^{42}$ Der Kurfürst und die Landesregierung konnten gemeinsam agieren, wobei hinzu kam, dass die Einsiedels als Inhaber altschriftsässiger Rittergüter ${ }^{43}$ Gerichtsstand direkt beim Landesherrn hatten. Durch Urteilseinholung an Spruchgremien und der damit zusammenhängenden Aktenversendung hatte sich in Kursachsen bereits seit dem 16. Jahrhundert ein Instanzenzug ausgebildet, der in diesem Fall indes umgangen wurde, indem sich Hildebrand der Ältere direkt an den Kurfürsten wandte. Obwohl Hildebrand von Einsiedel der Ältere als privater Kläger auftrat, wurde im Falle seines Sohnes ein Inquisitionsprozess ${ }^{44}$ geführt, dem nicht zuletzt ein herrschaftlicher Strafanspruch und der Anspruch auf Ausweitung der landesherrlichen Gerichtsbarkeit zugrunde lagen. Die Beziehungen zwischen Herrscher und Untertanen oblagen dabei einer zunehmenden Verrechtlichung; während der Kurfürst die Herrschaftspraxis bis ins 16. Jahrhundert verkörperte, verbreitete sich in der Folgezeit mehr und mehr eine institutionalisierte Form von Herrschaft, ohne dass damit jedoch die Person des Landesherrn als oberster Gerichtsherr obsolet geworden wäre.

Bezüglich der Kosten des Verfahrens hatte gleich zu Beginn Hildebrand der Ältere klar gemacht, dass er für alle anfallenden Kosten aufkäme bzw. diese aus dem Deputat seines Sohnes bestreiten würde. ${ }^{45}$ Auch die Geheimen Räte waren mit dieser Sache befasst; so bewilligten sie dem Sohn Hildebrand von Einsiedel die Korrespondenz an seinen Vater und den Kurfürsten. ${ }^{46}$

42 Im Gegensatz etwa zum in Leipzig angesiedelten Oberhofgericht, das in gewisser Konkurrenz zur Landesregierung stand. Seit dem 16. Jahrhundert hatte sich bei der Landesregierung eine Kompetenzverengung ergeben, um der schnell wachsenden Zahl von Justiz- und ,Policey'-Sachen Herr werden zu können; vgl. THOMAS KLEIN, Kursachsen, in: Kurt Jeserich/Hans Pohl/Georg-Christoph von Unruh (Hg.), Deutsche Verwaltungsgeschichte, Bd. 1: Vom Spätmittelalter bis zum Ende des Reiches, Stuttgart 1983, S. 810 f.

43 Der Unterschied zwischen Amts- und Schriftsassen betraf auch die Gerichtsbarkeit. Verfahren gegen Amtssassen wurden beim Amt geführt. Hinzu kam, dass bei schriftsässigen Adligen Kommissionen zum Einsatz kommen konnten; vgl. UlRIKE LUDWIG, Das Herz der Justitia. Gestaltungspotentiale territorialer Herrschaft in der Strafrechts- und Gnadenpraxis am Beispiel Kursachsens 1548-1648 (Konflikte und Kultur 16), Konstanz 2008, S. $42 \mathrm{f}$.

${ }^{44} \mathrm{Im}$ Gegensatz zum Akkusationsverfahren. Inquisitionsprozesse wurden an sich geführt, wenn kein privater Ankläger vorhanden war, was in diesem Fall jedoch nicht zutraf. Zum Verhältnis des Inquisitions- zum Akkusationsverfahren auch: LUDwIG, Justitia (wie Anm. 43), S. 62 f.

45 Hildebrand von Einsiedel der Ältere verglich sich mit dem Amtsschösser zum Hohnstein, Michael Böhme, bezüglich der Alimentierung seines Sohnes. In einem Schreiben vom 20. Mai 1638 aus Gnandstein schrieb Hildebrand, seinem Sohn solle von dessen deputierten Zinsen notdürftiger Unterhalt verordnet werden, dass der Kurfürst weder behelliget, angelaufen noch molestiret werden möge; vgl. HStA Dresden, Loc. 7193/6, fol. 30-32.

46 Der Amtsschösser von Hohnstein hatte gebeten, dass Hildebrand von Einsiedel gestattet werde, seinem Vater zu schreiben, den er um Verzeihung bitten wolle. Dies wurde von den Geheimen Räten bewilligt; vgl. HStA Dresden, Loc. 7193/6, fol. 45/4. Der Kurfürst gestattete den Schriftverkehr, wies indes aber den Schösser an, Hildebrands Post zu öffnen, den Inhalt zu lesen und in den Akten zu registrieren; vgl. HStA Dresden, Loc. 7193/6, fol. 129, 1. Oktober 1638. In der Folgezeit bat Hildebrand der Jüngere immer wieder um Feder, Tinte und Papier, was ihm zumeist gestattet wurde. 
Hildebrand von Einsiedel der Jüngere hingegen bemühte sich auf Hohnstein hingegen, seinen Lebenswandel auch in der Haft aufrecht zu erhalten. ${ }^{47}$ Ebenso war seine Haft zunächst auch von ständigen Supplikationen an seinen Vater geprägt, die sowohl auf Linderung der Haft als auch auf Freilassung gerichtet waren. ${ }^{48}$

Hildebrand von Einsiedel der Ältere bemühte sich dagegen, Zeugen aufzutreiben, um die gegen seinen Sohn erhobenen Vorwürfe beeiden zu lassen. Dabei spielte die Bezeugung der gegen ihn ausgestoßenen Verbalinjurien eine wichtige Rolle. ${ }^{49}$ Hildebrand von Einsiedel der Ältere scheute keine Mühen und finanzielle Mittel, um die Verfolgung des Prozesses auch weiterhin gewährleisten zu können. ${ }^{50}$

Das wegen Aktenversendung und der allgemeinen Kriegszeiten zeitraubende Verfahren brachte im April 1642 auch ein ,Bedenken` der Theologischen Fakultät der Universität Wittenberg hervor, welches das Rechtsverständnis Hildebrand von Einsiedels des Älteren eher antiquiert erscheinen ließ. ${ }^{51}$ Darin wird dem Kurfürsten geraten, Hildebrand den Jüngeren auf freien Fuß zu setzen. Dem Ermessen der Fakultät nach könne dem Sohn kein ewiges Gefängnis zuerkannt werden. Vielmehr seien väterliche und bewegliche Zurede und Anmahnungen zur Besserung viel kräftigere Mittel zu des Sohns Bekehrung.

Ganz allgemein wurden in schwierigen Fällen die Urteile auswärtiger Juristenfakultäten und Schöffenstühle eingeholt, diese Institutionen konnten dabei zu staatlichen Justizbehörden umfunktioniert werden. ${ }^{52} \mathrm{Da}$ der Fall Hildebrand von Einsiedels sich durchaus komplex darstellte, wurden die Urteile mehrerer Spruchkollegien eingeholt, deren Vergleich ein interessantes Licht auf das Rechtsempfinden und die Urteilpraxis verschiedener Territorien wirft. Das Urteil des Schöffenstuhls zu Halle befindet sich ganz auf der Linie des Vaters, wenn die Schöffen zu dem Schluss kommen: ad instan-

47 Der Kurfürst hatte u. a. angeordnet, dass sich der Verhaftete täglich mit vier Kannen Bier begnügen solle, bis seine Gläubiger befriedigt seien; vgl. HStA Dresden, Loc. 7193/7, fol. 9, 6. Juni 1638. Der Amtsschösser berichtete dagegen dem Kurfürsten, dass der Gefangene sechs Kannen Bier am Tag begebret, die er bei Gebrauchung des Tabaks austrinket; vgl. ebd., fol. $13 \mathrm{f}$.

48 Vgl. StA Leipzig, RG Gnandstein, Nr. 1294, Supplikationen Hildebrands an seinen Vater 1638-1640.

49 Vgl. StA Leipzig, RG Gnandstein, Nr. 1292, Zengnuß-Registratur Hildebrands des Eltern contra seinen zum Hohnstein gefenglicher Haft enthaltenen Sobn Hildebrand von Einsiedel (den Jüng.) kaiserl. Geschworene Notarien Anno 1640. Hildebrand von Einsiedel der Ältere bot vier Zeugen auf, die nach vorheriger Rechtsbelehrung am 20. April 1640 eidlich die in zwölf verschiedenen Punkten aufgeführten Vorwürfe des Vaters bestätigten. Zuvor hatte Hildebrand der Ältere noch einmal bekräftigt, sein Sohn wolle das eine oder andere Verbrechen nicht gestehen, deswegen sei er noch auf dem Hohnstein.

50 Dem kurfürstlichen Wunsch, Hildebrand möge zur Deckung der Unkosten 100 Taler zahlen (vgl. HStA Dresden, Loc. 7193/6, fol. 203 f., 28. Juli 1640), wurde entsprochen. Indes schrieb der Kurfürst noch vor Ablauf des Jahres erneut an Hildebrand, dass von dem Geld nichts mehr übrig sei und wegen Prozess und Winterkleidung noch etwas vorgeschossen werden solle, auch damit sich sein Sohn um so weniger zu beschweren habe; vgl. ebd., fol. 231, 15. Dezember 1640.

51 Vgl. Bedenken der Theologischen Fakultät der Universität Wittenberg vom 12. April 1642, HStA Dresden, Loc. 7193/6, fol. 323-328.

52 Vgl. KARL HÄRTER, Strafverfahren im frühneuzeitlichen Territorialstaat: Inquisition, Entscheidungsfindung, Supplikation, in: Blauert/Schwerhoff (Hg.), Kriminalitätsgeschichte (wie Anm. 39), S. 459-480, hier S. 465. Bei leichteren Delikten wie deviantem Lebenswandel konnte auch ein dualer Inquisitionsprozess erfolgen: ein lokales Untersuchungsverfahren und ein zentrales Entscheidungsverfahren. 
tiam Parentis zu surrogiren sprechen wir vor Recht, wiewol ein ewig Gefengnüß, als welches in Rechten der Todesstrafe gleichgeachtet wirdt, nicht stadt findet, so mag doch der Vater des ungehorsamen Sobns, gestalten sachen nach ... perpetuam custodiam ibme wol dictiren, und ist die Obrigkeit dieselbe zu exequiren schuldig, auch obne seinen willen zu mildern nicht befugt. ${ }^{53}$

Im Vergleich der Urteile nimmt die Juristische Fakultät der Universität Jena eine mittlere Stellung ein, indem sie für Recht erachtet, dass die oberzeblten Verbrechungen insgesambt, nicht also beschaffen, daß der Delinquent solcher wegen am Leben gestraft werden könnte, sondern es were derselbe uffs höchste mit dem Staupenschlag, oder nach gelegenheit seines Standes mit der ewigen Landesverweißung anzuseben, und zu belegen. ${ }^{54}$

Immerhin existiert hier ein Querverweis auf eine Rechtssprechung, die dem jeweiligen sozialen Stand der Delinquenten angepasst war. In beiden Fällen handelte es sich um Urteile auswärtiger Spruchkollegien, die indes interessante Vergleiche in der interterritorialen Spruchpraxis ermöglichen. Letztlich war jedoch die kursächsische Gerichtsverfassung maßgebend, was die Urteile zum Fall Hildebrand von Einsiedels anging. Die Juristische Fakultät der Universität Leipzig entsprach wohl am ehesten dem Rechtsempfinden des Kurfürsten, wenn sie zu dem Urteil gelangte: daß der verhafftete durch die in die vierthalb Jabr nunmehr erduldete continuirliche schwere Gefängnüß diese Verbrechungen ziemlichen verbüßet, auch, besorge der Acten, in solcher custodi in unterschiedenen Schreiben, so er an den Vater abgehen laßen, depreciret, umb verzeibung wehemütig angesucht ... So hat auch dabero nach gelegenheit dieser und anderer Umbstände die von dem Vater gesätzte Straffe perpetua custodiae nicht stadt, sondern es wird der verhaftete Hildebrand von Einsiedel auß Churfürstl. Hobeit und macht und mildigkeit der gefänglichen haft nunmebr billich hinwiederumb erlaßen, und auff frej̈en Fuß gestellet. ${ }^{55}$ Zusammen mit einem ernsten Verweis im Beisein seines Vaters und der Verpflichtung, eine öffentliche Abbitte zu leisten, konnte Hildebrand von Einsiedel der Jüngere damit rechnen, sich bald wieder auf freiem Fuß zu befinden.

Hildebrand von Einsiedel der Ältere appellierte jedoch - in Kenntnis dieser Rechtsprechung - wiederholt an den Kurfürsten, seinen Sohn in möglichst ewiger Haft zu belassen. ${ }^{56}$ Hildebrand der Jüngere, dem die Urteilssprüche in seinem Sinne nicht verborgen geblieben waren, bat hingegen den Kurfürsten um Ausspruch und Dezision sowie die Entscheidung zu fällen, das Urteil zu publizieren, demzufolge ihm das Gefängnis erlassen werden sollte. ${ }^{57}$

53 Urteilsspruch der Fürstlich Erzbischöflich Magdeburgischen Schöffen zu Halle; vgl. StA Leipzig, RG Gnandstein, Nr. 1296. Inwieweit dieser konservative Spruch im Sinne der Anschuldigungen des Vaters für dieses Spruchkollegium als typisch zu erachten ist, muss weiteren Untersuchungen vorbehalten bleiben. Festzuhalten bleibt, dass es sich hier um ein Spruchkollegium auf dem Gebiet eines geistlich verwalteten Erzstiftes handelte. Für Kursachsen sind die Spruchkollegien als Bestandteil der Gerichtsverfassung charakterisiert worden; vgl. HEINER LÜCK, Die kursächsische Gerichtsverfassung 1423-1550 (Forschungen zur deutschen Rechtsgeschichte 17), Köln/Weimar/Wien 1997.

54 Vgl. StA Leipzig, RG Gnandstein, Nr. 1296.

55 Urteil der Juristenfakultät der Universität Leipzig, Ende 1642; vgl. StA Leipzig, RG Gnandstein, Nr. 1294.

56 Z. B. ein Schreiben vom Juni 1642, in dem Hildebrand den Kurfürsten bittet, es bei ewiger custodia zu belassen, nachdem drei gleichförmige Urteile zugunsten des Verhafteten ausgefallen waren; vgl. HStA Dresden, Loc. 7193/6, fol. 355-369, 23. Juni 1642.

57 Vgl. HStA Dresden, Loc. 7193/6, fol. $371 \mathrm{f}$. 
Nachdem Hildebrand von Einsiedel der Ältere einen Termin in Dresden hatte verstreichen lassen, ${ }^{58}$ stellte der Kurfürst es der Direktion der Landesregierung anheim, darüber zu entscheiden, wie und wo Hildebrand von Einsiedel der Jüngere in Arrest verbleiben solle. 59

Schließlich wurde Hildebrand von Einsiedel der Jüngere am 15. April 1643 auf freien Fuß gesetzt, nicht ohne seinem Vater allerdings demütige Abbitte getan und seine Sünden bekannt zu haben. ${ }^{60}$ Ausschlaggebende Ursache für seine Freilassung war wohl nicht sein häufiges Supplizieren aus der Haft, sondern die juristische Spruchpraxis in seinem Sinne, die ein so hartnäckiges Anhalten seines Vaters um ewige Haft als rückwärtsgewandt erscheinen ließ. Mit der Anerkennung seiner Schuld hatte sich Hildebrand von Einsiedel der Jüngere den Status als gnadenwürdiger Delinquent verdient, sodass der Kurfürst nun auch die Tugend der Gnade walten lassen konnte. ${ }^{61}$

Dass ein Adliger auf Veranlassen seines eigenen Vaters fünf Jahre in Haft gehalten wurde, ist wohl einzigartig in der sächsischen Strafrechtsgeschichte, da Konflikte dieser Art eher familienintern geregelt wurden. In diesem Fall war es wohl ein allzu biblisch-theologisch fundiertes Verständnis vom strafenden Arm der Obrigkeit, wenn auf Anhalten Hildebrand von Einsiedels des Älteren auch Theologische Fakultäten von Universitäten mit dem delinquenten Verhalten seines Sohnes befasst wurden. Im Rechtsdenken des 17. Jahrhunderts gab es gerade in Kursachsen durchaus noch theokratisch-absolutistische Rechtsauffassungen. ${ }^{62}$ Insofern fügt sich das Rechtsempfinden Hildebrand von Einsiedels des Älteren ein in ein lutherisch geprägtes Obrigkeitsverständnis, das bei einer Strafzumessung auch biblische Argumente umfasste. Auf Ebene der europäischen Herrscherhäuser und Dynastien hingegen wurden theologische Fundierungen und Begründungen von Regierungshandlungen bis ins 19. Jahrhundert als fast unverzichtbares Mittel der Herrschaftslegitimation herangezogen;63

58 Hildebrand der Ältere entschuldigte sich beim Kurfürsten für sein Nichterscheinen mit den allgemeinen Kriegsumständen, weswegen er mehr Zeit brauche. Zum Zeitpunkt der Ladung stand er immerhin im 77. Lebensjahr; vgl. StA Leipzig, RG Gnandstein, Nr. 1297. 394.

59 Schreiben vom 7. April 1643 an die Regierung; vgl. HStA Dresden, Loc. 7193/6, fol.

60 Vgl. HStA Dresden, Loc. 7193/6, fol. 399-404.

61 Der Topos des gnädigen Herrschers spielte im Tugendkatalog frühneuzeitlicher Herrschererziehung und -inszenierung durchaus eine Rolle. Neben die Gerechtigkeit des Fürsten trat hier eine christlich inspirierte Gnadenpraxis, an die zu appellieren sich auch bei schwereren Delikten lohnte; vgl. LudwiG, Justitia (wie Anm. 43), S. 182 f. Im Fall von Verbalinjurien muss dagegen die Erfolgsquote von Suppliken als wenig aussichtsreich eingestuft werden; vgl. ebd., S. 243.

62 So Benedict Carpzov (1595-1666) als einer der führenden Juristen Kursachsens, dessen Rechtsempfinden dieser Richtung zuzuordnen ist. Er wurde auch als Ankläger in Hexenprozessen bekannt, er beriet den Kurfürsten Johann Georg I. bei der Abfassung seines Testaments und war von 1653 bis 1661 als Geheimer Rat in Dresden tätig. Ab 1633 war er Vorsitzender des Leipziger Schöffenstuhles. 1638 erschien von ihm das Handbuch des sächsischen Strafprozessrechts „Peinlicher sächsischer Inquisitions- und Achtsprozess“, das bis ins 18. Jahrhundert die deutsche Strafrechtswissenschaft prägte. Auch eine systematische Darstellung des protestantischen Kirchenrechts geht auf ihn zurück; vgl. zu seinem Leben und Werk: HeINER LÜCK, Benedict Carpzov (1595-1666) und der Leipziger Schöffenstuhl, in: Uwe Schirmer (Hg.), Sachsen im 17. Jahrhundert. Krise, Krieg und Neubeginn (Schriften der Rudolf-Kötzschke-Gesellschaft 5), Beucha 1998, S. 101-114.

63 Vor allem durch das sogenannte Gottesgnadentum; vgl. auch: ANDREAS PEčAR/KAI Trampedach (Hg.), Die Bibel als politisches Argument. Voraussetzungen und Folgen 
Argumente, die auf tiefer liegenden Ebenen der Gesellschaft im Zuge einer sich entwickelnden Strafrechtswissenschaft nicht ausschließlich zur Anwendung kommen konnten.

\section{Schluss und weiterfübrende Überlegungen}

Nachdem Hildebrand von Einsiedel der Ältere 1647 gestorben war, gingen die schriftsässigen Rittergüter Gnandstein und Wolftitz gemäß der Verfügungen seines Testaments an seinen Sohn Heinrich und seinen Enkel Haubold (1627-1687). Als 1649 auch Heinrich erbenlos starb, musste seine Witwe finanziell versorgt werden, was Hildebrand dem Jüngeren zufiel. Es erstaunt schon, dass Hildebrand als letzter überlebender Sohn Hildebrands des Älteren offenbar nach seinen Erfahrungen und Disziplinierungsmaßnahmen nach wie vor nicht in der Lage war, angemessen zu wirtschaften und mit seinen Mitteln hauszuhalten. Zunächst vertröstete er immer wieder die Witwe seines Bruders und konnte nur mit Hilfe seines Familiennetzwerkes unter Aufbietung aller Kräfte finanzielle Mittel bereitstellen. ${ }^{64}$ Curt von Einsiedel (1597-1668) 65 trat hier als eine Art Familienpatron auf, der sich um die Beschaffung der finanziellen Mittel kümmerte, es allerdings nicht verhindern konnte, dass zur Abtragung von Schulden das Rittergut Wolftitz unter Zwangssequester gestellt werden musste. Die Witwe Heinrich von Einsiedels wendete sich mehrfach an den Kurfürsten, um an ihre finanziellen Ansprüche durchzusetzen. Hildebrand von Einsiedel hingegen, der nach dem Tode seines Bruders das Gut Wolftitz übernommen hatte, vermochte es nicht ohne die intensive Hilfe seiner Verwandten, eine Klärung der Situation herbeizuführen. ${ }^{66} 1651$ gelang es Curt von Einsiedel, das Geld vorzustrecken und so den Rittergutsbesitz der Familie langfristig zu erhalten.

Festzuhalten bleibt noch das Ende Hildebrands des Jüngeren. Als er sich an Weihnachten 1652 zu Wolftitz vermählte, erkrankte er noch während der Hochzeitsfeierlichkeiten und starb, ohne Erben zu hinterlassen, am 4. Januar 1653. In der Familiengrablege zu Gnandstein ist er beerdigt worden. ${ }^{67}$

biblizistischer Herrschaftslegitimation in der Vormoderne (Historische Zeitschrift, Beihefte, NF 43), München 2007.

64 Vgl. StA Leipzig, Rittergut Wolftitz 1513-1937, Nr. 170, Briefe und persönliche Unterlagen Hildebrand von Einsiedels, unpag. (1649-1651), darin: Aufbringung $600 \mathrm{fl}$. darinnen die nechsten Agnaten consentiren wollen, zu befriedigung meines Bruders Seel. binterlaß. Fr. Witwen (darzu ist aller möglichst u. beste Fleiß angewendet worden).

651636 konnte er es sich leisten, den beharrlichen Wunsch des Kurfürsten, ihn zum Geheimrat zu bestallen, abschlägig zu bescheiden und stattdessen ab 1638 als hochfürstlichmagdeburgischer Geheimrat mit Dienstsitz in Halle in die Dienste von dessen Sohn August zu treten. Im Juli 1643 gewährte August, postulierter Erzbischof von Magdeburg, seinem Geheimrat ein Gnadengeld von 12.000 Talern; vgl. StA Leipzig, RG Gnandstein, U 120, Halle, 20. Juli 1643.

66 In einem Schreiben an seine Verwandten auch aus entfernteren Linien des Hauses Einsiedel berief sich Hildebrand von Einsiedel auf die Verfügung seines Vaters, dass ihm 20.000 Gulden Kapital gereicht werden sollen, wenn er ein Rittergut bezöge und käuflich erwürbe; vgl. StA Leipzig, Rittergut Wolftitz, Nr. 170, Schreiben vom 11. Juli 1650 an Einsiedel auf Scharffenstein, Dölnitz, Syhra, Prießnitz und Gnandstein.

67 Vgl. Valentin KÖNIG, Genealogische Adelshistorie, Bd. 1, Leipzig 1727, S. 276. In der halbseitigen Kurzbiografie ist mit keinem Wort vom unsteten Lebenswandel des Hildebrand von Einsiedels die Rede. Das Zerwürfnis mit seinem Vater und die fünfjährige Festungshaft werden diskret verschwiegen. So dienten Adelsgeschichten im 18. Jahrhun- 
Das Leben Hildebrand von Einsiedels und sein Lebensstil eignen sich bei allen Besonderheiten gut, den Standort der mittlerweile fest etablierten Kriminalitätsforschung im Umfeld der Bewältigung sozialer Konflikte zu verdeutlichen. Der Aufstieg der Forschung zur Delinquenz oder Devianz bewegt sich eher von zuweilen verengenden juristischen Normen weg, um zu einer möglichst breiten gesellschaftlichen Deutung zu kommen. So besaß Hildebrand von Einsiedel als Adliger ein hohes soziales Kapital, sodass sein Vater wohl nicht zuletzt dadurch gezwungen war, durch direkte Anrufung des Landesherrn eine Disziplinierung zu erreichen. Andererseits wurde dadurch die in Ansätzen bereits vorhandene Justiz- und Gerichtshierarchie unterlaufen, obwohl bei adligen Schriftsassen der Landesherr als oberste Instanz gefordert war.

In einem Punkt mutet das Verhalten Hildebrand von Einsiedels des Älteren hingegen geradezu modernistisch an. Die Intention, seinen Sohn mithilfe des Kurfürsten und seiner rechtlichen Sanktionsmöglichkeiten zu disziplinieren, führt in ihrer Argumentation immer wieder von deliktbezogenem Verhalten weg und zielt auf eine biografisch begründete Bestrafung, die die Ursachen für die Devianz und Delinquenz des Sohnes in seiner Mentalität erblickt: und will mir nicht gebühren, solcher vornebmer Collegiorum judicata weiter zu censiren, oder in zweiffel zu ziehen ... alß von bloßen jurisconsultis gesprochen, und weiter nicht passiren laßen, so findet sich darauß nichts anders, alß waß die Herren Concipienten, nur blo $\beta$ uff die delicta alleine, wie Sie in Actis befunden, ganz und gar aber nicht, uff das delinquentis totam vitam, impoenitentiam, et incorrigibilitatem, undt waß etwan in futurum, ferner vor scandala, von Ihme, sich zu befürchten, gesehen ... den ein Jurist beÿ seiner profession und pronunciation in Schöppenstüblen, und Facultäten, nicht weiter seben thut. Mit der Einführung des Biografischen in den Prozess und letztlich auch in das Strafmaß, das Hildebrand von Einsiedel der Ältere mit Blick auf seinen Sohn postuliert, konstituiert er damit den Kriminellen. Mit diesem Versuch, die determinierende Ursache für Devianz bzw. Delinquenz im Biografischen zu verorten, befindet er sich innerhalb von Deutungsmustern, in denen sich die Kriminologie heute noch befindet. ${ }^{68}$

Noch hatte Kursachsen auf dem Weg zu einem Rechtsstaat mit durchgestuften Gerichtshierarchien und nachvollziehbaren Entscheidungsfindungen eine lange Strecke zu bewältigen.

dert nicht zuletzt auch der Herrschaftslegitimation des Adels, in dem vermeintlich oder tatsächlich nicht standesgemäße Lebensabschnitte dem Leser verheimlicht wurden.

68 Vgl. dazu Michel Foucault, Überwachen und Strafen. Die Geburt des Gefängnisses, Paris 1975, S. 323 f. Die biografische Untersuchung als wesentliches Element des Gerichtsverfahrens macht das Leben des Delinquenten für seine Charakterisierung entscheidend und unterscheidet den Delinquenten damit vom „bloßen“ Rechtsbrecher. Der Konstituierung einer delinquenten Biografie kommt damit entscheidende Bedeutung zu bei der Entwicklung des modernen Strafvollzugswesens weg von den alten Leib- und Lebensstrafen. Unabhängig vom Verbrechen wird der „Kriminelle“ geschaffen. 